\title{
Meta
}

Journal des traducteurs

Translators' Journal

\section{HUMBLEY, John (2018) : La néologie terminologique. Limoges : \\ Lambert-Lucas, 472 p.}

\section{Bruno Courbon}

Volume 64, numéro 3, décembre 2019

URI : https://id.erudit.org/iderudit/1070546ar

DOI : https://doi.org/10.7202/1070546ar

Aller au sommaire du numéro

Éditeur(s)

Les Presses de l’Université de Montréal

ISSN

0026-0452 (imprimé)

1492-1421 (numérique)

Découvrir la revue

Citer ce compte rendu

Courbon, B. (2019). Compte rendu de [HuMbLEY, John (2018) : La néologie

terminologique. Limoges : Lambert-Lucas, 472 p.] Meta, 64(3), 832-836.

https://doi.org/10.7202/1070546ar

Ce document est protégé par la loi sur le droit d'auteur. L'utilisation des services d'Érudit (y compris la reproduction) est assujettie à sa politique d'utilisation que vous pouvez consulter en ligne.

https://apropos.erudit.org/fr/usagers/politique-dutilisation/
Cet article est diffusé et préservé par Érudit.

Érudit est un consortium interuniversitaire sans but lucratif composé de l'Université de Montréal, l'Université Laval et l'Université du Québec à Montréal. Il a pour mission la promotion et la valorisation de la recherche. https://www.erudit.org/fr/ 
Nous avons vu à travers notre étude que cette capacité hétérarchique de la langue se trouve adossée à une autre capacité de la langue, également centrale pour comprendre autant son évolution que celle de la linguistique: son homéostasie, c'est-à-dire sa capacité à garder son équilibre de fonctionnement dynamique malgré et au gré des évolutions constantes. (p. 232)

À la suite de la lecture du présent ouvrage, nous souhaitons formuler la conclusion suivante: il s'agit d'un ouvrage qui se fait remarquer par son originalité. Il est le résultat d'une expérience solide en terminologie, en traduction et en linguistique du corpus, mais aussi d'une lecture soigneuse confirmée par la riche bibliographie afférente. Mojca Pecman a réussi à offrir un éclairage nouveau sur certaines questions touchant à la langue et au discours spécialisés.

Héba Medhat-LecocQ INALCO, Paris, France

\section{RÉFÉRENCES}

Condamines, Anne et Rebeyrolles, Josette (1997): Point de vue en langue spécialisée. Meta. 42(1):174-184.

Culioli, Antoine (1997): À propos de la notion. In: Claude Rivière et Marie-Line Groussier, dir. La notion: actes $d u$ colloque "La notion». (Colloque «La notion», Paris, février 1996). Gap/Paris: Ophrys, 9-24.

Culioli, Antoine (1987/1990): La linguistique: de l'empirique au formel. In: Antoine Culioli. Pour une linguistique de l'énonciation: opérations et représentations. Vol. 1. Gap/Paris: Orphys, 9-46.

Halliday, Michael A. K. (1998/2004): Things and Relations: Regrammaticizing Experience as Technical Knowledge. In: Michael A. K. HALLIDAY. The Collected Works of M. A. K. Halliday. (Textes réunis par Johnathan J. Webster) Vol. 5. The Language of Science. Londres/New York: Continuum, 49-101.

Halliday, Michael A. K. (1995/2004): Language and the Reshaping of Human Experience. In: Michael A. K. Halliday. The Collected Works of M. A. K. Halliday. (Textes réunis par Johnathan J. Webster) Vol. 5. The Language of Science. Londres/New York: Continuum, 7-24.

Halliday, Michael A. K. (1961): Categories of the theory of grammar. Word. 17(3):241-292.

SAger, Juan C. (1997): Term formation. In: Sue Ellen Wright et Gerhard Budin, dir. Handbook of Terminology Management. Vol. 1. Amsterdam/Philadelphie: John Benjamins, 25-41.

SINCLAIR, John (2000): Lexical Grammar. Darbai ir dienos. 24:191-203.
Humbley, John (2018): La néologie terminologique. Limoges: Lambert-Lucas, $472 \mathrm{p}$.

Toute création langagière déplace, ne serait-ce qu'imperceptiblement, le regard porté sur les objets de représentation. Étudiée de longue date dans les domaines des arts et des lettres, elle retient depuis un bon demi-siècle l'attention de chercheurs qui s'intéressent aux termes des sciences et des techniques, qu'ils soient officiels ( $c f$. les usages recommandés) ou non (jargons de métiers, argots...). En 2017 paraissait, dans le onzième numéro de la revue Neologica, le texte des interventions de la journée d'étude TOTh tenue l'année précédente et intitulée La néologie en terminologie. Un an plus tard (en 2018), paraît un ouvrage au titre voisin, La néologie terminologique. Malgré l'ordre dicté par la chronologie des évènements, La néologie terminologique est le premier ouvrage qui offre un tour d'horizon détaillé du sujet. Il s'agit d'une somme érudite. L'auteur, John Humbley, œuvre depuis le début des années 1970 dans les domaines présentés, à la fois comme acteur et comme chercheur mû par la volonté d'allier la théorisation à un travail d'analyse de corpus. Sont exposés et discutés dans ce livre une large sélection d'options théoriques, de résultats de travaux empiriques et de mises en œuvre pratiques. L'auteur met de plus à l'épreuve des faits trois des modèles qu'il juge les plus pertinents pour appréhender les phénomènes de la néologie en terminologie. Il précise qu'une revue exhaustive des travaux en la matière était impossible, mais ne cache pas l'ambition encyclopédique de son entreprise (p. 395; aussi, p. 207 : "présenter autant de travaux que possible»), ce qui ressort de la bibliographie: avec 44 pages et une quinzaine de références par page, environ 700 textes y sont présentés, écrits en français, en allemand, en anglais, en italien, en portugais, en danois... (le cas échéant, l'auteur traduit lui-même les extraits qu'il cite); de nombreuses références sont discutées sous divers angles, à différentes étapes du développement.

Les lecteurs pourront aussi découvrir l'amorce d'une synthèse instrumentale, puisque l'auteur propose le prototype d'un modèle d'analyse original, multidimensionnel, de la néonymie, à partir duquel il examine, entre autres, des cas de rétronymie, d'éponymie, d'emprunt, de «glissement de sens", de composition, de synonymie. À la suite de Louis Guilbert, Humbley insiste sur l'importance du foisonnement synonymique dans la phase de création terminologique (processus fréquent dans la création d'usages "généraux»). Il présente alors (p. 174-175) les cas inverses, d'une part de différenciation de synonymes (en citant le cas de synchronisation et alignement en traduction automatique), et d'autre part, de neutralisation 
d'une distinction sémantique (cas de vinyle, utilisé aujourd'hui pour parler - sans doute par rétronymie sémantique - de disques "non compacts", incluant désormais les 78 tours). L'auteur nomme «modèle mixte» le modèle qu'il propose - bien qu'il s'en défende, jugeant "prématuré de proposer un modèle en l'absence de chantier néologique important» (p. 122). Celui-ci combine trois grands types d'approche qui aident à mieux saisir la création de nouveaux termes (approches «incrémentale», «textuelle» et "cognitive»). La multidimensionnalité consistante de ce modèle rend en grande partie compte de la complexité des phénomènes recouverts par le terme néologie terminologique.

Comme l'expose l'auteur, cette formule recouvre des phénomènes et des enjeux de natures diverses qu'il serait réducteur de considérer sous un seul angle. Situant son travail dans un mouvement dialectique entre la production d'une vision panoramique - l'objectif étant de mieux comprendre les enjeux et les motivations reliés à la création de nouveaux termes - et l'élaboration d'outils d'analyse dont la fabrication s'appuie sur les résultats de l'examen de cas concrets (des "échantillons", dans les termes de l'auteur), Humbley montre bien à quel point c'est plutôt un paquet de questions que soulève une réflexion approfondie sur les phénomènes de néologie terminologique. Entre autres, on y retrouve le nécessaire besoin de (re)définir la ligne de partage entre synchronie et diachronie - donc entre "l'ancien» et «le nouveau» (l'auteur rappelle que l'identification de la néologie présuppose une perspective diachronique [p. 42]; v. aussi Humbley 2006) -, entre variabilité des usages effectifs et évolution des normes de référence, entre usage spécialisé et usage général, entre le concret du discours, le formel de la langue et l'abstrait du concept... Ce paquet de questions méritait un temps suffisant pour être constitué et n'être pas envoyé de façon (trop) expéditive, mais aussi pour que commencent à être élaborées des réponses développées et cohérentes, à la lumière des connaissances et des outils actuels (l'auteur rappelle, justement, qu'il manque «une vision d'ensemble» [p. 206]). L'un des grands mérites de l'ouvrage est ainsi de rassembler et de créer des liens entre des constellations de réflexions théoriques, des travaux empiriques et des mises en œuvre pratiques, qui, trop souvent, sont restés dans l'ignorance de leur existence respective.

La préoccupation récurrente pour la création de termes spécialisés a régulièrement été prise pour objet de réflexion, mais, hormis dans les travaux pionniers d'un défricheur comme Louis Guilbert (entre autres, [Guilbert 1975]), cette réflexion a très rarement, ces dernières décennies, donné lieu à un développement qui lui soit entièrement consacré (l'ouvrage de Jean-François Sablayrolles, La néologie en français contemporain, qui portait sur les usages néologiques dans le lexique général, ne fait que très ponctuellement mention de la néologie spécialisée [Sablayrolles 2000: 131]). Le livre de Humbley comble donc manifestement une lacune. On saisit bien, à sa lecture, les attentes, tant théoriques que méthodologiques et pratiques, auxquelles répond un questionnement à propos des phénomènes de création terminologique. L'ouvrage répond également à un besoin de synthèse historique des travaux produits dans une période particulièrement «néologène» (terme repris [BC] de Gardin [1974: 69]; entre les années 1960 et les années 2010, la production et la circulation d'objets, de procédés et d'actions, notamment, n'a cessé de croître, rendant cruciale la création de quantité de termes... et leur compréhension).

La néologie terminologique invite à (se) poser des questions à propos des multiples dimensions constitutives du geste terminogène. Dépassant le cadre, utilement circonscrit en son sein, de la création de termes dans des domaines de spécialité - qu'ils soient professionnels, scientifiques ou techniques -, ces questions paraissent aussi simples qu'elles sont complexes en réalité: elles portent sur la "conscience» de la nouveauté terminologique (ou de la nouveauté de la chose exprimée par celle-ci; on [BC] pourrait discuter l'idée, associée à Croft et surtout reprise de Keller, selon laquelle, dans la langue générale, "l'évolution se fait sans que les locuteurs s'en rendent compte» [p. 86]), sur les conditions de son partage, et sur la pluralité des formes et des valeurs sémantiques, mais aussi rhétoriques, voire idéologiques, qu'elle prend dans les diverses communautés qui les produisent, les ré-utilisent et contribuent ainsi - ou non - à les diffuser. Mais l'ouvrage aborde également la question des limites formelles des signes-dénominations. Autrement dit: qu'est-ce qu'un terme? Ou, plus spécifiquement: peut-on se satisfaire de l'importation, pour des faits de lexique, d'une approche syntaxique classique, fondée sur le découpage morphocatégoriel de la phrase en parties du discours pour en dégager des segments dénominatifs? (Humbley [2012] rappelait que la dénomination est au cœur de la pratique terminologique; il entérine ici ce point de vue [p. 133]: "[les] liens entre concept et dénomination » étant le «véritable locus de la néonymie» - et souligne les limites d'une approche centrée sur le terme seul -, c'est-à-dire, pris en dehors de ses contextes discursif et conceptuel [p. 34].) Ces questions présentent aussi l'intérêt de nourrir la réflexion quant à la pertinence et aux limites des procédés métalinguistiques utilisés pour tâcher de mieux comprendre la production de termes nouveaux: effet d'une perspective sémasiologique ou "onomasiologique» sur le 
traitement de ce type de faits linguistiques - mais cette dernière est-elle réellement possible d'un point de vue analytique, sachant qu'une analyse rétrospective comporte le biais de la connaissance a posteriori de ce qui n'était, au moment de l'acte de création, qu'un futur inconnu, comme une Kann-Norm, si l'on pastiche Wüster? (BC); prise en compte de l'inscription des nouveaux termes dans des réseaux de relations conceptuelles et morphologiques établis, mais aussi considération pour la «fonction» qu'ils remplissent au sein des milieux dans lesquels ils sont amenés à circuler (conditions sociocognitives: les exigences ne sont pas les mêmes, que les termes soient partagés par des microspécialistes, par des connaisseurs d'un domaine ou par des «profanes» - l'auteur consacre une partie du développement aux phénomènes de néologie de transfert, interlinguistique, mais aussi intralinguistique, vulgarisation comprise); recours à une méthodologie fondée sur l'analyse de textes (concret des réalisations) et/ou sur l'analyse de concepts et de leur structuration (abstraction des idées).

La néologie terminologique et la méthode proposée pour l'étudier sont examinées, illustrées et mises à l'épreuve des faits au moyen de plusieurs études de cas développées, réalisées par l'auteur au fil des années, ou reprises d'autres auteurs; car il ne s'agit pas seulement «d'analyser et de rendre compte de l'évolution » manifestée dans les emplois néologiques, mais également de la "documenter» (p. 204), en exploitant un corpus diversifié (écrits des «inventeurs», articles de revues spécialisées et de journaux de grande diffusion, dictionnaires...). L'auteur présente alors les résultats d'analyses qu'il a menées sur la «mise en place» du vocabulaire de l'enregistrement sonore au XIX ${ }^{\mathrm{e}}$ siècle, ainsi que sur la création terminologique dans les domaines de la nouvelle économie et du commerce électronique à la fin du $\mathrm{xx}^{\mathrm{e}}$ siècle. En très bon connaisseur des objets et des domaines concernés (objets: la néologie, les langues de spécialité, les phénomènes d'emprunt interlinguistiques et intersectoriels/ interdisciplinaires, le passage d'un usage spécialisé vers des usages généraux...; domaines: la terminologie - avec, entre autres, une discussion serrée des préceptes, hérités ou questionnés, de la terminologie générale d'Eugen Wüster ${ }^{1}$-, la lexicologie - dont le terreau fertile de la lexicologie historique française des années 1950-1970 dans laquelle œuvrèrent notamment Georges Matoré, Peter J. Wexler, Louis Guilbert et Bernard Quemada -, mais également la linguistique générale - avec en particulier l'exploitation de propositions théoriques de M. A. K. Halliday, de George Lakoff, de William Croft et de Vincent Nyckees), l'auteur consacre un bon quart de l'ouvrage (une centaine de pages) à dresser un panorama (le parcours de lecture est sportif!) des productions métanéonymiques des cinq dernières décennies (à propos de formulation, on pourra noter une variation terminologique ici: néologie terminologique ou néologie en terminologie, néologie spécialisée, création/formation de termes et néonymie sont le plus souvent pris comme des équivalents - à propos de ce dernier terme, Jean-Claude Boulanger informait les lecteurs du Français moderne que c'est à Guy Rondeau, auquel Humbley attribue aussi la paternité du terme, que l'on devait ce néonyme ${ }^{2}$ [Boulanger 1981: 382]). L'auteur présente et discute les positions et propositions de trois générations de chercheurs et de praticiens, en partant de l'origine de l'institutionnalisation des domaines (disciplinarisation de la lexicologie et de la terminologie au sein des établissements universitaires et scientifiques) et de la constitution d'un corps d'études aujourd'hui clairement identifiées sous l'appellation "la néologie» (la revue Neologica, fondée à la fin des années 2000 par John Humbley et Jean-François Sablayrolles, illustre la fertilité de ce champ de recherche, qui, il y a une génération, existait davantage sur le plan de la description terminographique, par l'entremise de revues comme La banque des mots ou La clé des mots en France, et Néologie en marche au Québec).

Humbley rappelle les liens étroits entre terminologie et néologie, d'une part, et politiques d'aménagement (ou de planification) linguistique et corps constitués pour les mettre en œuvre, d'autre part. Il consacre un chapitre entier à la «néologie officielle», distinguant le plan de la normalisation technoscientifique du plan de l'action politique. Sont alors présentés le travail de concertation et de diffusion terminologiques des Commissions ministérielles (contexte français), ainsi que le rôle moteur de l'Office [devenu Office québécois] de la langue française. Mais Humbley offre encore un aperçu «extra-francophone» de ce qu'il appelle "la néologie terminologique politique» (l'accent est mis sur les réseaux au sein des communautés ibériques et scandinaves). Il met également en relief l'effet catalyseur entraîné par le renouveau des outils (grandes banques de données, logiciels spécifiques...) et des façons de procéder (term mining, veille technologique, dépouillement automatisé - incluant l'acquisition et la reconnaissance de termes).

Mais la plus grande originalité de l'ouvrage tient au choix de combiner trois modèles théoriques provenant d'horizons différents. Prudent, l'auteur les présente comme des «hypothèses» (p. 159, 341) qui restent encore au stade de "postulats" (p. 119). Intégrés dans le modèle de création de concepts scientifiques du chercheur allemand Holger Becker, ces trois modèles se rapportent chacun à une dimension particulière des faits de néologie 
terminologique: le «modèle incrémental», inspiré des travaux du linguiste japonais Kyo Kageura, est pertinent pour décrire la formation des termes, considérés sous l'aspect morphosémantique de leur expansion, incluant la réduction sémiotique (en l'illustrant à partir du cas emblématique de la composition, Humbley situe ce modèle «à un niveau avant tout descriptif» [p. 159]); le "modèle discursif », inspiré des travaux du linguiste australien d'adoption M. A. K. Halliday, aide à saisir le potentiel d'émergence terminologique qui a cours dans la relative fluidité de l'expression discursive, les concepts connexes d' "emballage» (packing) et de «métaphore grammaticale» proposés par Halliday, qui décrivent des formes particulières de figement linguistique, s'avèrent utiles en néoterminologie (on peut se demander si le recours aux deux autres métafonctions - idéationnelle et interpersonnelle - que proposait Halliday ne présenterait pas un intérêt pour examiner les choix de formulation opérés par les sujets à l'origine de nouveaux termes); enfin, inspiré du transfert effectué par la linguiste belge Rita Temmerman de concepts de la linguistique cognitive vers le domaine de la terminologie, le «modèle cognitiviste» ou «modèle métaphorique» est utilisé, avec notamment l'idée de "modèles cognitifs idéalisés» (Idealized Cognitive Models, concept proposé par Lakoff en 1987), pour faire état des modes de conceptualisation sous-jacents facilitant l'intégration de nouveaux usages terminologiques (que ceux-ci aillent de pair avec la création de termes nouveaux ou qu'ils passent par des formes existantes - ce que Gabrielle Quemada [1983: x] nommait la «néologie passive»). Bien qu’un crédit soit donné, dans l'ouvrage, à l'approche continuiste développée par Vincent Nyckees en sémantique diachronique (Nyckees 2006), il n'est pas certain que le point de vue cognitiviste (objectiviste et nettement a-sémiotique et a-subjectif) de Lakoff et celui, médiationniste et continuiste (nettement sémiotique) de Nyckees, puissent, sur le fond théorique, être combinés, hormis - et c'est peut-être l'une des forces heuristiques de l'assemblage «œcuménique» $(\mathrm{BC})$ entrepris dans cet ouvrage - s'ils sont placés sur des plans différents (ce qu'indique l'auteur lorsqu'il présente les composantes du «modèle mixte» [p. 119]).

La démarche suivie par l'auteur dans cet ouvrage est à l'image de la façon dont il se propose d'appréhender son objet: 1) approche rétrospective (le chapitre 8 est entièrement consacré à la présentation - illustrée - d'une méthode en «néologie historique spécialisée» qui consiste à remonter à «l'ancien», à «l'existant» [p. 97, 341], c'est-à-dire à «ce» à partir de et par rapport à quoi les nouveaux termes ont été produits); 2) compréhension aussi large que possible de la question en (re)donnant la parole aux principaux acteurs (chercheurs/inventeurs du passé et contemporains) qui l'ont abordée; 3) mise en valeur critique de la diversité des points de vue en jeu, sans enfermement doctrinaire.

Une prise en considération sérieuse des subjectivités cognitives, et, en l'occurrence, des effets, à l'échelle collective, des compétences relativement singulières des sujets parlants sur les productions lexicales, demeure sans doute plus taboue encore dans les domaines scientifiques et techniques où prime la quête d'une certaine objectivité et où, par conséquent, on part du principe qu'il peut exister une terminologie fixe et comprise de tous (les nomenclatures de certains domaines comme la chimie - voir p. 241 et suiv. - se rapprochent sans doute de cet idéal, mais qu'en est-il de la vaste majorité des usages terminologiques?). En faisant état des travaux qui traitent du résultat de la néologie terminologique (les «néologismes»), et en insistant sur l'importance de considérer les conditions extra- ou intersubjectives, les plus formalisables, du processus de néologie, Humbley suggère, çà et là, qu'il sera pertinent, pour que l'analyse rétrospective des phénomènes néoterminologiques soit complète, de faire aussi porter l'attention sur les conditions (inter)subjectives de la création de termes, y compris chez les spécialistes (dont l'accès aux connaissances spécialisées - et à la connaissance des termes d'un domaine - peut varier, dont la compréhension des concepts est plus ou moins précise, dont l'inscription dans une «école» peut orienter le regard - donc déterminer la signification associée aux termes -, mais également, et en premier lieu, dont l'intérêt plus ou moins marqué pour certains sujets tend à renforcer ou à diminuer d'une certaine façon les fondements intuitifs). Une approche psychoterminologique de la genèse des termes scientifiques et techniques reste à bâtir.

Par son apport historique et théorico-méthodologique, le livre de Humbley contribuera certainement à orienter la réflexion et les pratiques de demain. Espérons que son appel à la création d'un forum regroupant les recherches sur la question (p. 397) sera entendu. Ce livre pourra servir d'ouvrage de référence à des étudiants avancés, et sera très utile aux chercheurs qui s'intéressent de près à la terminologie, à la linguistique diachronique et à l'histoire des usages linguistiques, mais également aux historiens de la linguistique, et, plus largement, aux historiens des sciences et des techniques ${ }^{3}$, ainsi qu'aux épistémologues intéressés par les faits de langue, car, comme le fait remarquer l'auteur, on peut envisager la néologie terminologique « comme un révélateur de la nouveauté dans les découvertes scientifiques et techniques» (p. 30).

Bruno Courbon Université Laval, Québec, Québec, Canada 


\section{NOTES}

1. Comme le note l'auteur, la néologie est peu conceptualisée dans les pays où l'héritage wüstérien est plus direct; la faible considération pour la dimension diachronique semble en être la raison principale: «[...] on peut considérer qu'il existe un hiatus entre la théorie de Wüster, pour qui la terminologie est complètement synchronique, et le postulat de la néonymie, qui est par définition diachronique, voire panchronique» (Guilbert 1973: 26).

2. «Les familles lexicales semblant avoir horreur du vide, quelque linguiste avisé créera, si ce n’est déjà fait, le néographe nécessaire à néologue. [...] 500 MN [500 mots nouveaux définis et expliqués, par Jacques Cellard et Micheline Sommant, 1979, dont J.-Cl. Boulanger faisait alors le compte rendu] propose le terme néonyme, qui tendrait à remplacer néologisme! [sic] [...] [R]appelons que G. Rondeau a proposé dans un récent colloque sur la terminologie (Moscou, novembre 1979) le terme néonymie pour désigner les néologismes techniques et scientifiques» (Boulanger 1981: 382). John Humbley fait état (p. 35) de divers termes forgés pour parler de néonymes (dont néoterminisme - proposé par Alain Rey [Rey 1979 - référence non citée dans la bibliographie], repris ponctuellement par l'école fonctionnelle de Martinet -, et néonyme). Il fait l'hypothèse que le terme néoterminologie, employé par Julie Pelletier et Andy Van Drom - alors étudiants-chercheurs à l'Université Laval -, aurait pu être inspiré par la norme ISO 1087-1: 2000. Il est plus probable que le néonyme néoterme soit repris de Jean-Claude Boulanger, qui en faisait usage dès le début des années 1980, en lui associant une nuance qui le distingue de son proche voisin néonyme: "On nous permettra une remarque supplémentaire à propos de néonymie et du dérivé néonyme. Le raffinement terminologique et sémantique peut tendre vers une telle perfection que certains objecteront que néonymie et néonyme ne sont pas satisfaisants. Étymologiquement néonymie et néonyme incluent nom, mot et terme (ou terminologisme). Il serait peut-être plus juste d'utiliser néoterme ou néoterminologisme plutôt que néonyme lorsqu'il est question de terminologie?» (Boulanger 1983: 319 - un peu plus tôt dans ce texte, Boulanger propose aussi néonymiste pour parler des acteurs du domaine [Boulanger 1983: 315]; voir aussi Boulanger 1989). Néoterme était, chez JeanClaude Boulanger, le pendant, pour les usages spécialisés, de néomot.

3. Ce dont l'auteur est bien conscient: «Les historiens des sciences ne se trompent pas lorsqu'ils se penchent sur les questions de terminologie dans le but de comprendre les évolutions scientifiques» (p. 27).

\section{RÉFÉRENCES}

Boulanger, Jean-Claude (1981): Compte rendu de 500 mots nouveaux définis et expliqués par Jacques Cellard et Micheline Sommant [1979, Paris/Gembloux: Duculot]. Le français moderne. 49(1):379-382.

Boulanger, Jean-Claude (1983) : Synonymie, néonymie et normalisation en terminologie. Commentaire d'un exposé d'Alain Rey. In: Diane Duquet-Picard et Marian BugaraAdshead, dir. Problèmes de la définition et de la synonymie en terminologie. (Colloque international de terminologie, Québec, 23-27 mai 1982). Québec: Girsterm, 311-327.

Boulanger, Jean-Claude (1989): L'évolution du concept de néologie de la linguistique aux industries de la langue. In: Caroline De Schaetzen, dir. Terminologie diachronique. (Colloque «Terminologie diachronique, Bruxelles, 25-26 mars 1988). Paris: Conseil international de la langue française/Ministère de la communauté française de Belgique, 193-211.

GARDIn, Bernard (1974): La néologie, aspects sociolinguistiques. Langages. 36:67-73.

Guilbert, Louis (1975): La créativité lexicale. Paris: Larousse.

Humbley, John (1993): L'observation de la néologie terminologique. La banque des mots. 5:65-74.

Humbley, John (2006): La néologie: interface entre ancien et nouveau. In: Rosalind GREENSTEIN, dir. Langues et cultures: une histoire d'interface. Paris: Publications de la Sorbonne, 93-103.

Humbley, John (2012): Retour aux origines de la terminologie: l'acte de dénomination. Langue française. 174:111-129.

Rey, Alain (1979): La terminologie. Paris: Presses universitaires de France.

Quemada, Gabrielle, dir. (1983): Dictionnaire de termes nouveaux des sciences et des techniques. Paris: Conseil international de la langue française.

Sablayrolles, Jean-François (2000): La néologie en français contemporain. Paris: Honoré Champion.

Kurdi, Mohamed Zakaria (2018): Traitement automatique des langues et linguistique informatique. Vol. 2. Sémantique, discours et applications. Sciences cognitives. Londres: ISTE Éditions, $323 \mathrm{p}$.

Dans son ouvrage, Mohamed Zakaria Kurdi, professeur au Département d'informatique du Lynchburg College (Virginie, États-Unis), s'applique à dresser la deuxième partie de son «bilan panoramique» (p. 12) du traitement automatique des langues (TAL), comme lui-même l'avance, un effort qui est couronné de succès en février 